\title{
Characterizations of solute binding proteins by DSF scanning and crystallography
}

\author{
Umesh Yadava $^{1}$, Matthew W. Vetting ${ }^{2}$, Steven C. Almo ${ }^{2}$ \\ ${ }^{1}$ Department Of Physics, DDU Gorakhpur University, Gorakhpur, INDIA, Gorakhpur, India, ${ }^{2}$ Department of Biochemistry, Albert \\ Einstein College of Medicine, New York, United States \\ E-mail: u_yadava@yahoo.com
}

The solute binding proteins (SBP) are responsible for the capture of ligands in the periplasm, and subsequent transfer of these solutes to integral membrane transporters for passage in to the cytoplasm to meet their ultimate fate. The importance of SBPs for functional assignments cannot be overstated, as the first reactant for many/all catabolic pathways must be imported from the extracellular milieu via a transport system. Thus the ability to identify the initial reactant bound to SBP places enormous constraints on the regions of chemical space which need to be considered for computational and experimental efforts, and provides immediate insight in to in vivo functions and the metabolic contribution of entire pathways. The discovered liganded SBPs structures have provided critical conformation of ligand binding preferences and contributed to our understanding of new metabolism1. Furthermore, liganded SBP structures are of particular importance, as only by observing the experimentally determined pose of the ligand in the binding site can the precise determinants responsible for recognition be identified. These determinants provided strong constraints for predicting specificity boundaries i.e. where in sequence space homologs begin to recognize additional and /or different ligands/substrates. This information is also essential for defining the confidence limits associated with annotations made by sequence comparisons and serve as a powerful tool for the selection of new targets predicted to possess novel specificities.

In the present study, the structural and functional characterizations of several solute binding proteins have been carried out. Proteins were cloned from genomic DNA, expressed by autoinduction, and purified by a combination of Ni-NTA and size exclusion chromatography. The purified SBPs were screened using differential scanning fluorometry (DSF) and a $>400$ compound ligand library. Two of the SBPs exhibited DSF hits that were novel for their respective transport family2. Crystallization trials of proteins have been conducted with their respective DSF ligand hits. Those SBPs that have structures determined and their respective interactions with co-crystallized ligands will also be presented.

1. Zhao, S. et al. (2013). Nature, 502, 698-702.

2. Yadava, U. et al. (2016) Acta Cryst., F72, 467-472.

Keywords: Solute binding Proteins, Differential Scanning Flourimetry, Functional annotation 